\title{
SISTEMAS DINÁMICOS DISCRETOS Y UN POCO DE CAOS
}

\section{DISCRETE DYNAMICAL SYSTEMS AND CHAOS}

\author{
Roberto Ávila-Pozos ${ }^{1}$, Raúl Temoltzi-Ávila ${ }^{2}$
}

\section{Resumen}

Presentamos una breve introducción a los modelos matemáticos discretos. Estos modelos se pueden emplear para representar el comportamiento de sistemas biológicos, bajo ciertas suposiciones.

Se muestra el caso de una ecuación en diferencias lineal de orden uno y su riqueza dinámica. Con esta ecuación, representamos el caso del crecimiento de una población con recursos ilimitados, por lo que su comportamiento es de crecimiento ilimitado.

También se presenta una ecuación en diferencias no lineal de orden uno. Esta ecuación sirve para modelar el crecimiento de una población cuando existe una capacidad límite para que el medio pueda albergar a dicha población. Esta ecuación se conoce como ecuación lógistica. Es de enorme interés notar cómo, al modificar uno solo de los parámetros de la ecuación, se genera un comportamiento caótico.

Palabras clave: modelos matemáticos, tasa de crecimiento, bifurcación

\section{Abstract}

We present a brief introduction to discrete mathematical models. This models are use to explain the behavoir on biological systems, under a set of certain conditions.

We show a linear difference equation and its no ordinary dynamic. With this equation, we modeling population growth with unlimited resources so this population has an unlimited growing.

A non linear difference equation is showed too. This equation is used to modeling growing in a population where exist a limiting capability in order to support this population. This model is called logistic equation. In this case, we could see how modifying a single parameter, it is generated a chaotic behavior.

Keywords: Mathematical Modeling, growth rate, bifurcation

\footnotetext{
${ }^{1}$ Roberto Ávila-Pozos. Profesor Investigador del Área Académica de Matemáticas y Física. Doctor en Ciencias por la Universidad Autónoma Metropolitana, México. Sus temas de investigación son Modelos en células excitables, epidemiología matemática y dinámica de poblaciones. Autor de correspondencia. Correo-e: ravila@uaeh.edu.mx

${ }^{2}$ Raúl Temoltzi-Ávila. Profesor Investigador del Área Académica de Matemáticas y Física. Doctor en Ciencias por la Benemérita Universidad Autónoma de Puebla, México. Sus temas de investigación están relacionados con la Teoría de Control.
} 


\section{INTRODUCCIÓN}

Los modelos matemáticos se han usado desde hace mucho tiempo para explicar fenómenos biológicos, como el propuesto por Thomas Malthus para explicar el crecimiento de la población. El uso de modelos teóricos para describir el comportamiento de sistemas biológicos es muy útil para examinar problemas de gran escala. Los modelos matemáticos pueden ayudar a validar teorías existentes o para proveer el conocimeinto necesario para describir los mecanismos mediante los cuales ocurren ciertos procesos.

La modelación matemáticas se puede utilizar para predecir el comportamiento de cierto fenómeno bajo determinadas condiciones, para generar experimentos virtuales, para comparar hipótesis, o para sugerir conjeturas o nuevas hipótesis.

El lenguaje matemático proporciona descripciones compactas de ideas complicadas y debería ser capaz de ofrecer conocimiento. Los modelos matemáticos no son perfectos; en el mejor de los casos, son buenas aproximaciones a la realidad, que nos ayudan a entender un poco más sobre el comportamiento de sistemas complejos.

Los modelos matemáticos discretos, se refieren a modelos de tiempo discreto. Estos modelos miden, por ejemplo, el tamaño de una población en intervalos regulares y fijos de tiempo. Esta aproximación es muy útil para sistemas que cambian en distintas generaciones, o en los casos en los que los datos están disponibles sólo en intervalos regulares de tiempo. Estos modelos se componen con ecuaciones en diferencias.

Muchos organismos tienen nacimientos, muertes y otros procesos demográficos que ocurren en intervalos regulares. Por ejemplo, los programas de manejo y captura de ciertas especies, se realizan de manera regular, formando ciclos discretos de tiempo.

El cambio en el tamaño de la población puede calcularse si se conocen las tasas de nacimiento, de defunción y de migración. Una población cerrada, no tiene migración, es decir, su tamaño cambia solamente a través de los nacimientos y decesos, y el cambio en el tamaño de la población depende únicamente de las tasas de nacimiento y defunción.

La formulación de un modelo específico requiere suposiciones explícitas sobre las tasas de natalidad

y mortandad. Idealmente estas suposiciones se hacen con el objeto de responder aspectos biológicos de interés.

En el caso de microorganismos, es razonable asumir que el nacimiento de nuevos organismos es proporcional al número de organismos presente. En términos matemáticos, si el tamaño de la población al tiempo $n$ es $x_{n}$, entonces, después de un intervalo de tiempo, el número de nacimientos es una fracción del tamaño de la población $b x$, para alguna constante $b$ llamada tasa de nacimiento per capita. Similarmente, se puede asumir que el número de decesos en el mismo intervalo de tiempo es una fracción del tamaño de la población $d x_{n}$, para alguna constante $d$, la tasa de mortalidad per capita. Por lo tanto, el cambio neto en el tamaño de la población es una relación entre las fracciones de nacimientos y defunciones en cada intervalo de tiempo.

La razón de cambio de alguna cantidad, con frecuencia es proporcional a la cantidad actual. Esto puede ser cierto en el caso de una población que no tiene restricción en el crecimiento. Sea $x_{n}$ el número de miembros de la población en el tiempo $n$ y sea $r$ la tasa de crecimiento per cápita de la población. La ecuación en diferencias que describe el crecimiento de la población es

$$
x_{n+1}=r x_{n}
$$

Una solución de la ecuación en diferencias (1) es una expresión que proporciona el valor de $x$ para todos los valores de $n$. Si conocemos el tamaño inicial de la población, $x_{0}$, entonces podemos calcular 


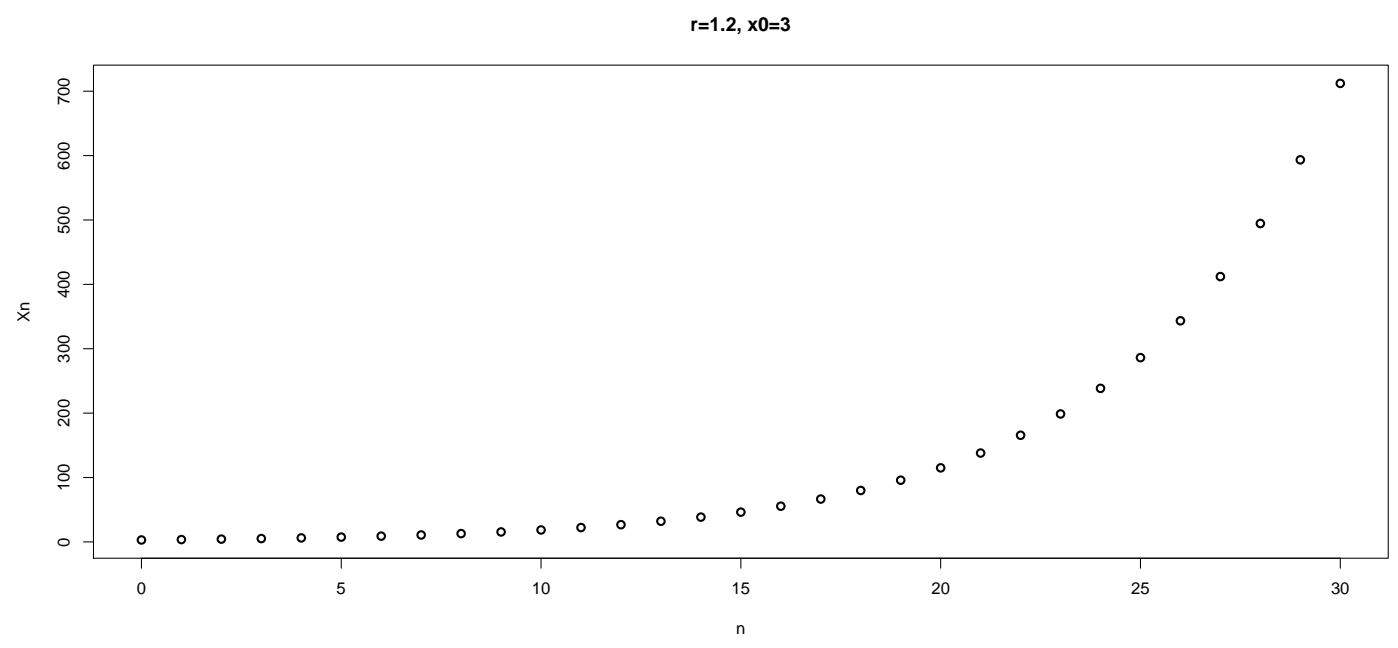

Figura 1: Solución de la ecuación (1), con $r=1.2$ y $x_{0}=3$.

$x_{1}, x_{2}, \ldots, x_{n}$ de manera recursiva:

$$
\begin{aligned}
& x_{0} \\
& x_{1}=r x_{0} \\
& x_{2}=r x_{1}=r\left(r x_{0}\right)=r^{2} x_{0} \\
& x_{3}=r x_{2}=r\left(r^{2} x_{0}\right)=r^{3} x_{0} \\
& \vdots \\
& x_{n}=r^{n} x_{0}
\end{aligned}
$$

La especificación del valor de $x_{0}$ se llama condición inicial. La gráfica de la solución es el conjunto discreto de puntos $\left(n, x_{n}\right)$, como se muestra en la Figura 1.

Note que si $r>1$ el número de nacimientos es mayor que el número de defunciones, y la población crecerá exponencialmente. Si $r<1$, el número de decesos es mayor que el de nacimientos y la población decrece hasta desaparecer. Si $r=1$ la población permanece constante en el valor $x_{0}$.

Las ecuaciones en diferencias como la ecuación (1), se pueden escribir en forma general como

$$
x_{n+1}=r x_{n}+b
$$

que se clasifican como ecuaciones en diferencias lineales de orden uno. Si el valor de $b$ es cero, tenemos el caso de una ecuación en diferencias lineal homogénea de orden uno, como en el caso de la ecuación (1).

La ecuación (2), aunque no es muy diferente de la ecuación (1), tiene comportamientos distintos para diferentes valores de $r$.

Si $r=1$, entonces, la solución de la ecuación (2) es $x_{n}=x_{0}+n r$ y por lo tanto, la población crece sin límite si $n$ crece sin límite, cuando $b \neq 0$. Cuando $b=0$, la solución es $x_{n}=x_{0}$ para todos los valores de $n$.

Si $r \neq 1$, la solución es $x_{n}=\left(x_{0}-\alpha\right) r^{n}+\alpha$, con $\alpha=\frac{b}{1-r}$.

En este caso, si $|r|<1$, la población se aproxima al equilibrio $\alpha$, cuando $n$ crece sin límite (a menos que $\left.x_{0}=\alpha\right)$. 

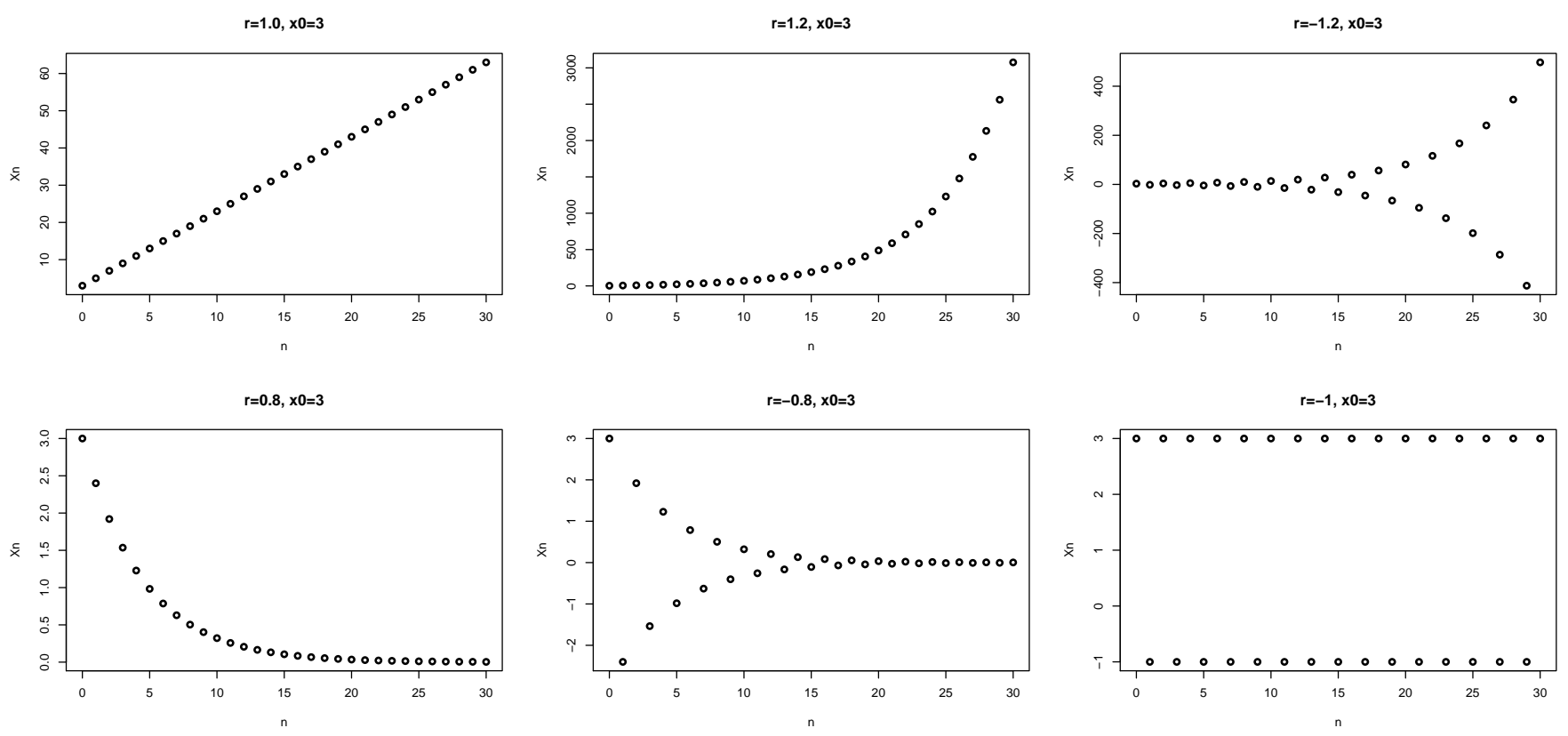

Figura 2: Soluciones de la ecuación (2) para distintos valores de $r$, con $x_{0}=3$.

Un equilibrio de la ecuación en diferencias $x_{n+1}=f\left(x_{n}\right)$ es la solución de la ecuación $x=f(x)$.

Si $|r|>1$, la población crece (o decrece) sin límite cuando $n$ crece sin límite (dependiendo del signo del parámetro $r$, como puede verse en la Figura 2).

Finalmente, si $r=-1$, la población oscila entre $x_{0}$ y $b-x_{0}$.

En la Figura 2 se muestran posibles comportamientos de la ecuación (2).

\section{UN MODELO DISCRETO DE CRECIMIENTO}

El modelo anterior supone una tasa de crecimiento constante. Esta suposición es poco probable en poblaciones reales, aunque podría ser razonable para micro-organismos. Para organismos más complicados, esta suposición es una sobre-simplificación, puesto que se ignora la competencia por los recursos, así como otros factores relevantes, como la estructura por edades (por ejemplo, la tasa de mortalidad puede depender más de la edad que de la densidad poblacional, mientras que la tasa de nacimientos dependerá más de la población reproductiva que del tamaño total de la población). Además, se debe considerar la posibilidad de que las tasas de natalidad y mortalidad pueden estar influenciadas por el tamaño de las especies que interactúan con la población bajo estudio (competencia, depredación, mutualismo).

Un modelo ampliamente estudiado es el de la ecuación logística. Esta es una ecuación en diferencias no lineal de orden uno:

$$
x_{n+1}=r x_{n}\left(1-\frac{x_{n}}{A}\right)
$$

donde $A$ se conoce como capacidad de carga de la población, que representa el tamaño de la población que puede soportarse con los recursos disponibles. El parámetro $r$ es la tasa intrínseca de crecimiento, y representa la tasa de crecimiento per cápita, cuando el tamaño de la población es lo suficientemente pequeño para asegurar que las limitaciones por recursos son despreciables. 
En este modelo, si $A \gg x_{n}$, el modelo se aproxima al descrito en la ecuación (1).

El modelo logístico predice un rápido comportamiento inicial para $0<x_{0}<A$; después, disminuye el crecimiento conforme pasa el tiempo, hasta que el tamaño de la población se aproxima a un valor límite. Este comportamiento se ha observado en algunas poblaciones, y por esta razón se emplea para describir la dinámica de una población.

Se puede demostrar que la ecuación (3) tiene dos equilibrios: $x_{\infty}=0$ y $x_{\infty}=2-r$. Se puede demostrar también, que si $1<r<3$, el equilibrio $x_{\infty}=0$ es inestable, y que el equilibrio $x_{\infty}=2-r$ es asintóticamente estable, y cuando $r>3$ ninguno de los equilibrios es estable.

\section{Y AHORA, CAOS}

¿Qué comportamiento tiene un sistema modelado con una ecuación en diferencias (ec. 3) que no tiene equilibrios estables?

¿Qué le ocurrirá a una población cuya tasa de crecimiento es muy grande entre una generación y otra?

En la primera generación, los miembros de la población, que tienen recursos suficientes, producen una segunda generación, que seguirá contando con recursos suficientes. Si la población presenta competencia, como en el modelo logístico, los miembros de la segunda generación no tendrán la misma cantidad de recursos que aquellos que tuvieron los de la primera generación. Entonces, la tercera generación será muy pequeña, pues en la segunda generación, pocos miembros habrán podido reproducirse. La tercera generación tendrá nuevamente muchos recursos y podrá reproducirse en gran número, así que la cuarta generación volverá a ser grande. Entonces, la población estará oscilando entre una generación grande y una pequeña. Se puede ver una pequeña oscilación en el modelo logístico cuando $2<r<3$; después, la población se aproxima a un estado intermedio. Se podría conjeturar que una población con una tasa intrínseca de reproducción grande puede causar fluctuaciones tales que la población no alcanza a establecerse. Eso es precisamente lo que pasa cuando $r>3$.

Cuando $r>3$, aparece una solución de periodo 2 en el modelo logístico. Esto significa que hay un par de valores $x^{+}$y $x^{-}$tales que $\operatorname{rg}\left(x^{+}\right)=x^{-}$y que $\operatorname{rg}\left(x^{-}\right)=x^{+}$. Entonces, la secuencia alternante $\left\{x^{+}, x^{-}, x^{+}, x^{-}, \ldots\right\}$ es una solución de la ecuación en diferencias (3). Puesto que la solución se repite después de dos términos, decimos que la solución es de periodo 2. Se puede verificar este comportamiento y encontrar los valores de $x^{+}$y $x^{-}$numéricamente o analíticamente.

Si se quieren encontrar los valores de $x^{+}$y $x^{-}$analíticamente, se debe resolver la ecuación $x_{n+2}=x_{n}$, es decir $\operatorname{rg}(\operatorname{rg}(x))=x$. Como la función $g(x)$ es una cuadrática, $\operatorname{rg}(\operatorname{rg}(x))=x$ es un polinomio de grado cuatro. Conocemos dos de las raíces del polinomio, $x=0$ y $x=A\left(1-\frac{1}{r}\right)$, por lo que la ecuación de grado cuatro se puede reducir a

$$
x^{2}-A\left(\frac{r+1}{r}\right) x+A^{2}\left(\frac{r+1}{r}\right)=0,
$$

cuyas raíces son

$$
x^{+}=\frac{A}{2 r}\left[r+1+\sqrt{r^{2}-2 r-3}\right], \quad x^{-}=\frac{A}{2 r}\left[r+1-\sqrt{r^{2}-2 r-3}\right] .
$$

La ecuación $r^{2}-2 r-3=(r-3)(r+1) \geq 0$, por lo tanto, puesto que $r>0$, cuando $r>3$ la ecuación en diferencias logística tiene una solución periódica de periodo 2 .

Para $r>3.4495$, la solución de periodo 2 es inestable, pero es posible probar que aparece una solución de periodo 4 y que esta solución es asintóticamente estable si $3.44495<r<3.544$. Cuando la solución 

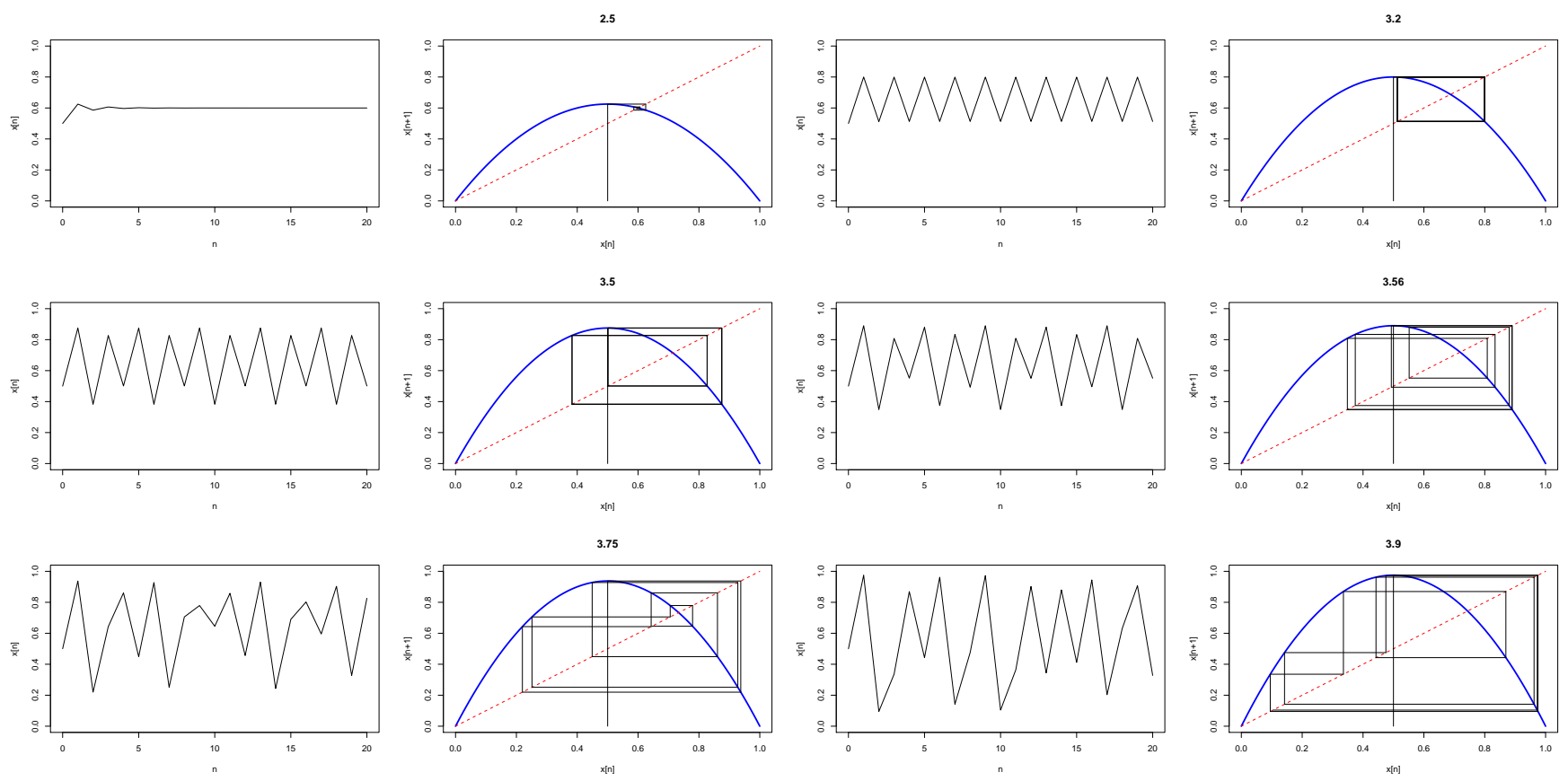

Figura 3: Comportamiento de la población y su respectivo diagrama de telaraña para diferentes valores de $r$ en la ecuación (3).

de periodo 4 se vuelve inestable, aparece una solución de periodo 8, y dicha solución es asintóticamente estable si $3.544<r<3.564$. Este fenómeno de doblamiento de periodo continúa hasta que $r=3.57$, cuando la solución periódica deja de tener periodos que son potencias de 2 . Para $r=3.75$, hay una solución de periodo 5. Algunos de estos comportamientos se presentan en la Figura 3.

Para cualquier entero positivo $p$, hay algún valor $r>3.828$ para el cual la ecuación (3) tiene una solución periódica de periodo $p$, pero diferentes valores iniciales dan soluciones cuyo comportamiento es muy diferente.

A este comportamiento se le conoce como caos.

Una representación de este comportamiento se ilustra con un diagrama de bifurcación en la Figura 4.

\section{CONCLUSIONES}

Se han presentado dos ejemplos de ecuaciones en diferencias de orden uno. Ambos modelos sirven para estudiar la dinámica de una población bajo supuestos diferentes.

En el caso de la ecuación en diferencias lineal, los recursos son ilimitados y los miembros de la población no tienen que competir entre ellos para sobrevivir y continuar reproduciéndose. En este ejemplo, se pueden estudiar dinámicas diferentes, únicamente modificando el valor del parámetro $r$. Este comportamiento se justifica plenamente mediante un teorema $[1,2]$.

En el caso de la ecuación en diferencias no lineal de orden uno, el crecimiento de la población depende de la cantidad de recursos. Esa condición se incorpora mediante el parámetro denominado capacidad de carga. Si la capacidad de carga es mucho mayor que el tamaño de la población, el crecimiento de esta población es similar al descrito en la ecuación (1).

Al ser una ecuación en diferencias no lineal, se puede realizar una linealización, y encontrar los 


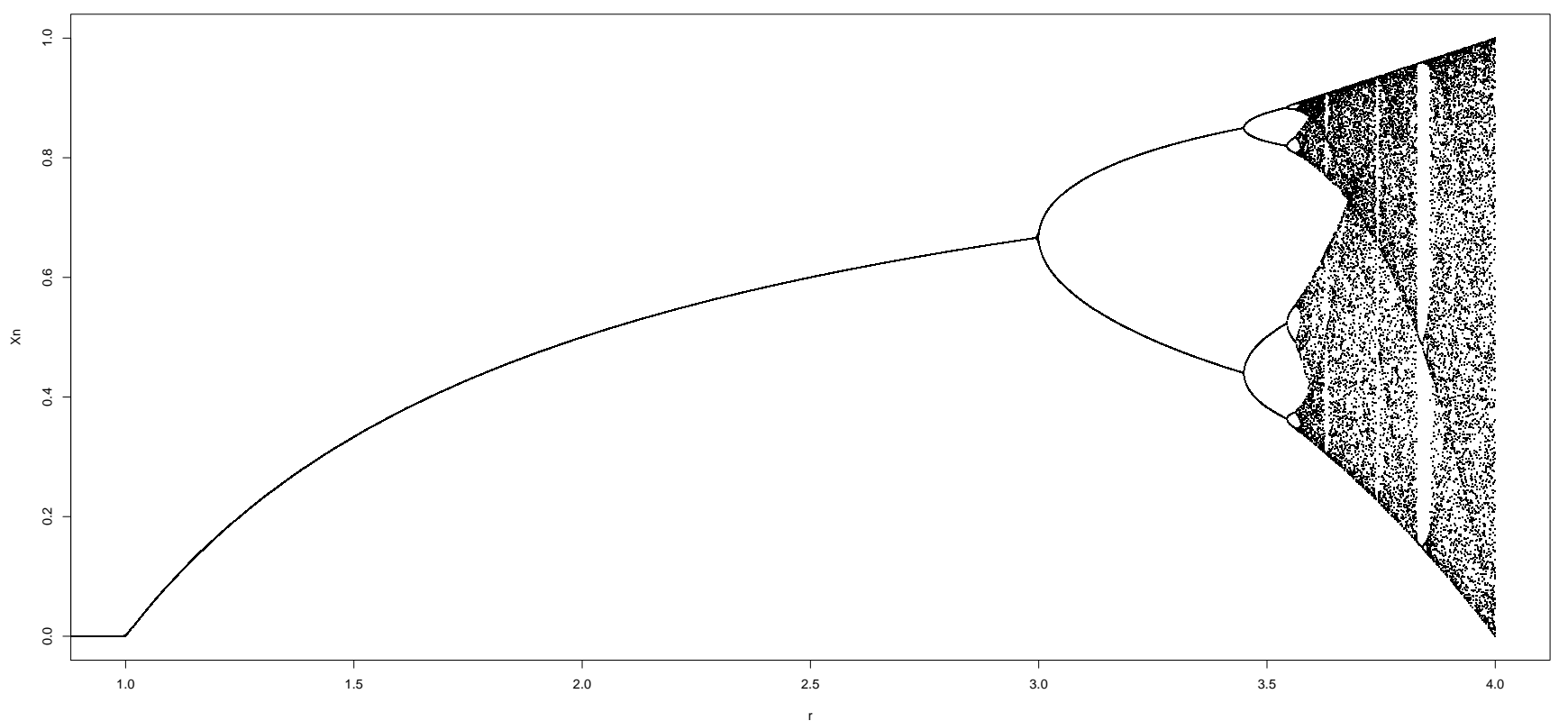

Figura 4: Diagrama de bifurcación generado con la ecuación (3).

puntos de equilibrio de la ecuación, y explorar su comportamiento. Se puede demostrar [3, 4, 5] la estabilidad de los puntos de equilibrio y se puede observar que esta ecuación de orden uno puede generar un comportamiento caótico al cambiar únicamente el parámetro $r$.

Resulta interesante remarcar cómo ecuaciones en diferencias, en apariencia sencillas, pueden generar comportamientos tan complejos como los mostrados en este trabajo (Figura 4).

También es importante mencionar que hay muchos resultados teóricos detrás de los modelos dinámicos discretos, y que este trabajo podría motivar al estudio de las ecuaciones en diferencias y a los sistemas de ecuaciones en diferencias.

\section{REFERENCIAS}

[1] F. Brauer and C. Castillo-Chavez, Mathematical Models in Population Biology and Epidemiology, Springer, New York, 2013.

[2] F. Brauer and Ch. Kribs, Dynamical Systems for Biological Modeling, CRC Press, New York, 2016.

[3] N. Britton, Essential Mathematical Biology,Springer, New York, 2012.

[4] J. Müller and Ch. Kuttler, Methods and Models in Mathematical Biology, Springer, New York, 2015.

[5] R. Murray, Mathematical Biology. 1 An Introduction, Springer, New York, 2011. 